[Article]

\title{
一种吡嗪铱(III)配合物的晶体结构及光物理性质
}

\author{
韦传东 ${ }^{1}$ 葛国平 ${ }^{1, *}$ 李春艳 ${ }^{1} \quad$ 雷克微 $^{1} \quad$ 梁洪泽 $^{1} \quad$ 禹 钢 $^{2} \quad$ 刘志伟 ${ }^{2, *}$ \\ ( ${ }^{1}$ 宁波大学材料科学与化学工程学院, 宁波市新型功能材料及其制备科学实验室-省部共建国家重点实验室培育基地, \\ 浙江 宁波 315211; 2 北京大学化学与分子工程学院, 稀土材料化学与应用国家重点实验室, 北京 100871)
}

\begin{abstract}
摘要：合成了一种铱配合物二(4,4'-二氟-5-甲基-2,3-二苯基吡嗪) (乙酰丙酮)合铱 $[(M D P P F) 2 \operatorname{Ir}(\operatorname{acac})]$ 的有机 电致发光器件(OLED), 利用 X射线单晶衍射仪测定了该化合物的晶体结构. 利用紫外-可见吸收光谱、发射光谱 对其光物理性质进行研究. 结果表明: (MDPPF $)_{2} \operatorname{Ir}(\mathrm{acac})$ 的单晶结构属于三斜晶系, $P \overline{1}$ 空间群, 晶胞参数 $a=$ 1.13984(3) nm, $b=1.26718(3) \mathrm{nm}, c=1.29541(3) \mathrm{nm}, \alpha=93.7181(19)^{\circ}, \beta=101.638(2)^{\circ}, \gamma=110.853(3)^{\circ}, V=$ 1.69336(7) $\mathrm{nm}^{3}$; (MDPPF $)_{2} \operatorname{lr}(\mathrm{acac})$ 在二氯甲烷溶液中的发射峰为 $555 \mathrm{~nm}$. 以(MDPPF $)_{2} \operatorname{Ir}(\mathrm{acac})$ 为客体材料, 制备了结构为 ITO/NPB(40 nm)/CBP: (MDPPF $)_{2} / r(a c a c)(20 \mathrm{~nm}) / \mathrm{TPBi}(10 \mathrm{~nm}) / \mathrm{Alq}_{3}(30 \mathrm{~nm}) / \mathrm{LiF}(1 \mathrm{~nm}) / \mathrm{Al}$ $(100 \mathrm{~nm})$ 的一系列不同掺杂浓度器件, 器件的发射峰位于 $558 \mathrm{~nm}$, 最大亮度达到 $32700 \mathrm{~cd} \cdot \mathrm{m}^{-2}$, 最大电流效率 $44.3 \mathrm{~cd} \cdot \mathrm{A}^{-1}$, 最大功率效率 $20.7 \mathrm{Im} \cdot \mathrm{W}^{-1}$.
\end{abstract}

关键词: 铱(III)配合物; 吡嗪; 磷光材料; 有机电致发光器件

中图分类号: 0641

\section{Crystal Structure and Photophysical Properties of an Iridium(III) Pyrazine Complex}

\author{
WEI Chuan-Dong ${ }^{1} \quad$ GE Guo-Ping, ${ }^{1, *} \quad$ LI Chun-Yan $^{1} \quad$ LEI Ke-Wei \\ LIANG Hong-Ze ${ }^{1} \quad$ YU Gang ${ }^{2} \quad$ LIU Zhi-Wei ${ }^{2, *}$
}

('State Key Laboratory Base of Novel Functional Materials and Preparation Science, Faculty of Materials Science and Chemical Engineering, Ningbo University, Ningbo 315211, Zhejiang Province, P. R. China; ${ }^{2}$ State Key Laboratory of Rare Earth Materials Chemistry and Applications, College of Chemistry and Molecular Engineering, Peking University, Beijing 100871, P. R. China)

\begin{abstract}
An iridium(III) complex of (MDPPF) 2 Ir(acac) (MDPPF=4,4'-difluoro-5-methyl-2,3-diphenylpyrazine, acac=acetylacetone) has been prepared and characterized by X-ray single crystal diffraction. The photophysical properties of (MDPPF) $)_{2} \operatorname{Ir}(\mathrm{acac})$ were investigated using UV-Vis spectroscopy and photoluminescence (PL) techniques. X-ray diffraction (XRD) studies revealed that the complex belongs to a triclinic system of the $P \overline{1}$ space group with cell dimensions $a=1.13984(3) \mathrm{nm}, b=1.26718(3) \mathrm{nm}, c=1.29541(3) \mathrm{nm}, \alpha=93.7181(19)^{\circ}, \beta=$ $101.638(2)^{\circ}, y=110.853(3)^{\circ}$ and $V=1.69336(7) \mathrm{nm}^{3}$. A solution of the (MDPPF) $2 \mathrm{r}(\mathrm{acac})$ complex in dichloromethane was found to emit yellow light with a maximum peak at $555 \mathrm{~nm}$ at room temperature. Furthermore, (MDPPF) $)_{2} \operatorname{Ir}(\mathrm{acac})$ was used as a phosphorescence dopant in organic light-emitting diodes (OLEDs) with the structures of ITO/NPB $(40 \mathrm{~nm}) / \mathrm{CBP}:(M D P P F)_{2} \operatorname{Ir}(\mathrm{acac})(20 \mathrm{~nm}) / \mathrm{TPBi}(10 \mathrm{~nm}) / \mathrm{Alq}_{3}(30 \mathrm{~nm}) / \mathrm{LiF}(1 \mathrm{~nm}) / \mathrm{Al}(100 \mathrm{~nm})$. These devices showed yellow emission at $558 \mathrm{~nm}$, a maximum brightness of $32700 \mathrm{~cd} \cdot \mathrm{m}^{-2}$, current efficiency of $44.7 \mathrm{~cd} \cdot \mathrm{A}^{-1}$ and power efficiency of $20.7 \mathrm{Im} \cdot \mathrm{W}^{-1}$.
\end{abstract}

Received: October 23, 2014; Revised: November 21, 2014; Published on Web: November 21, 2014.

"Corresponding authors. GE Guo-Ping, Email: geguoping@nbu.edu.cn; Tel: +86-574-87608784. LIU Zhi-Wei, Email: zwliu@pku.edu.cn; Tel: +86-10-62757156

The project was supported by the Ningbo Municipal Natural Science Foundation, China (2010A610164,2010A610187), Ningbo Social Development Project, China (2012C5002), and K. C. Wong Magna Fund of Ningbo University, China.

宁波市自然科学基金(2010A610164,2010A610187), 宁波市重大择优委托项目(2012C5002)和宁波大学王宽城基金资助

(c) Editorial office of Acta Physico-Chimica Sinica 
Key Words: Iridium(III) complex; Pyrazine; Phosphorescent material; Organic light-emitting diode

\section{1 引言}

自从 Forrest 等 ${ }^{1}$ 报道了第一个高效的电致磷光 材料八乙基卟啉铂(PtOEP)以来, 研究和开发新型的 电致磷光材料引起人们极大兴趣. 有机电致磷光材 料常用的重金属原子多为第 VIII 族的金属, 如锇 $(\mathrm{Os}) 、{ }^{2-5}$ 铂 $(\mathrm{Pt}) 、{ }^{6-9}$ 铱 $(\mathrm{Ir})^{10-16}$ 等, 由于它们强烈的自旋 轨道耦合, 使得其配合物的单线态激子和三线态激 子混杂, 三线态具有某些单线态特征, 三线态激子 的对称性被破坏, 缩短了磷光寿命, 减少了磷光猝 灭, 增强了单线态到三线态之间的系间窝跃和磷光 效率. 其中金属铱配合物具有相对较短的三线态寿 命和更高的发光效率, 能在室温下发出较强的磷 光, 是研究得最多的一种磷光材料. ${ }^{14}$ 铱配合物的激 发态包括金属与配体之间的能量传递(MLCT)态, 配 体与配体之间的能量传递(LLCT)态和配体内部的 能量传递(ILCT)态等, ${ }^{17-19}$ 使得重金属铱配合物的发 射波长、强度以及电化学性能会随着配体结构的改 变而发生明显的变化. ${ }^{20}$ 通过选择合适的配体(包括 $\mathrm{C}^{\wedge} \mathrm{N}$ 型环金属配体和辅助配体), 金属铱配合物已实 现可见光全波段范围的发光. ${ }^{14,21}$

吡嗪铱(III)配合物是一类优良的电致磷光材 料, 在有机电致磷光器件中有优异表现. 2003 年 Cheng 等 ${ }^{22}$ 报道了基于吡嗪铱配合物 $\operatorname{Ir}(\mathrm{DBQ})_{2}(\mathrm{acac})$ 和 $\operatorname{Ir}(\mathrm{MDQ})_{2}$ (acac) 的电致发光器件, 外量子效率分 别达到 $11.9 \%$ 和 $12.4 \%$. Bian 等 ${ }^{23}$ 制备的以铱配合物 $\operatorname{Ir}(\mathrm{DPQ})_{2}(\mathrm{CBDK})$ 为发光层的无掺杂器件的最大外 量子效率为 $6.2 \%$, 最大流明效率为 $3.49 \mathrm{~lm} \cdot \mathrm{W}^{-1}$. Ge 等 ${ }^{24}$ 报道的吡嗪铱配合物(MPPZ) $2 \mathrm{Ir}(\mathrm{acac})$, 其最大外 量子效率为 $13.2 \%$, 最大电流效率为 $37.3 \mathrm{~cd} \cdot \mathrm{A}^{-1}$, 最大 流明效率为 $20.3 \mathrm{~lm} \cdot \mathrm{W}^{-1}$. 张国林等 ${ }^{25,26}$ 报道了一系列 吡嗪铱配合物, 基于(DPP) $2 \operatorname{Ir}(\mathrm{acac})$ 和(DPQ) $2 \operatorname{Ir}(\mathrm{acac})$ 的电致发光器件的外量子效率分别达到 $13.85 \%$ 和 $5.14 \%$; 基于 (MDPP) $)_{2} \operatorname{Ir}(\mathrm{acac})^{27,28}$ 的器件的外量子效 率为 $6.02 \%$, 流明效率为 $9.89 \mathrm{~lm} \cdot \mathrm{W}^{-1}$, 亮度为 78924 $\mathrm{cd} \cdot \mathrm{m}^{-2}$. 但总的来说, 目前文献上报道的吡嗪铱配合 物磷光材料还不多.

据文献 ${ }^{29,30}$ 报道, 在分子内引入 $\mathrm{C}-\mathrm{F}$ 键能够减 小非辐射失活的速率, 改变分子堆积, 减少自淬灭 几率, 从而显著增加了分子的发光量子效率. 在本 文中, 通过在 $(\mathrm{MDPP})_{2} \operatorname{Ir}(\mathrm{acac})$ 的主配体上引入 $\mathrm{F}$, 得 到一种吡嗪铱(III)配合物(MDPPF) $2 \operatorname{Ir}(\mathrm{acac})$, 报道了
其晶体结构及电致发光性能.

\section{2 实验部分}

\section{1 仪 器}

晶体结构采用 Rigaku Mercury 70 CCD 型 X 射 线衍射(XRD)仪测试. 紫外-可见(UV-Vis)吸收光谱 采用 Shimadzu UV-2550 型紫外-可见光谱仪测定, 样品浓度为 $1 \times 10^{-5} \mathrm{~mol} \cdot \mathrm{L}^{-1}$. 光致发光 $(\mathrm{PL})$ 光谱由 Hitachi F-4600 荧光光谱仪测定. 电致发光(EL)器件 的电致发光光谱采用 Ocean Optics 2000 光谱仪测 量. 电流-电压-亮度曲线由 R6145(Advantest) 电压 计、 multimeter 2000(Keithley) 电流计和 LS-110(Minolta)亮度计测定.

\section{2 配合物(MDPPF) $2 \operatorname{Ir}(\mathrm{acac})$ 的晶体结构测定}

按文献 ${ }^{31}$ 方法合成配合物(MDPPF $)_{2} \operatorname{Ir}(\mathrm{acac})$. 以 二氯甲烷-乙醇为溶剂, 配制适量(MDPPF $)_{2} \operatorname{Ir}(\mathrm{acac})$ 配 合物的饱和溶液, 在室温下缓慢挥发, 约四周析出桔 黄色单柱形晶体, 选择尺寸为 $0.41 \mathrm{~mm} \times 0.23 \mathrm{~mm} \times$ $0.12 \mathrm{~mm}$ 大小的晶体用于结构测定. 在 293(2) K 温 度下, 在 Rigaku Mercury 70 CCD X 射线单晶衍射 仪上收集衍射数据, 共收集独立衍射点 6921 个. 用 SHELXS-97 程序完成结构解析, 用 SHELXL-97程 序完成结构精修. 对于所有非氢原子采用各向异性 和全矩阵最小二乘法进行修正.

\section{3 结果与讨论}

\section{1 晶体结构的描述}

单晶结构测定的结果表明, 配合物晶体属于三 斜晶系, $P \overline{1}$ 空间群, $R=0.0239, w R=0.0599$. 配合物主 要的晶体学数据见表 1 , 主要键长、键角见表 2 , 晶体 结构见图 1.

从表 2 可以看出, 所有键长和键角都在正常值 范围之内, 其中, $\mathrm{Ir}-\mathrm{C}$ 键长分别为 1.985(3)、1.989(3) $\mathrm{nm} ; \mathrm{Ir}-\mathrm{N}$ 键长分别为 2.017(2) nm、2.024(2) nm, 略 长于 $\mathrm{Ir}-\mathrm{C}$ 键. $\mathrm{O}-\mathrm{Ir}-\mathrm{O}$ 键角为 $87.85(8)^{\circ} ; \mathrm{C}-\mathrm{Ir}-\mathrm{O}$ 键角为 $175.05(10)^{\circ} ; \mathrm{N}-\mathrm{Ir}-\mathrm{C}$ 键角为 $121.5(3)^{\circ} ; \mathrm{N}-$ $\mathrm{Ir}-\mathrm{O}$ 键角分别为 $95.17(9)^{\circ} 、 84.87(9)^{\circ}$, 其键角的变 化范围较大, 此外, 从椭球图可知, 分子具有中心对 称性, 另一个重要特征是环金属配体上的吡嗪环和 两个苯环平面的二面角分别为 $13.78^{\circ} 、 44.4^{\circ}$.

从图 1 的晶体结构图中可以看出, 铱原子与其 
表 1 (MDPPF) $\operatorname{Ir}(\mathbf{a c a c})$ 的晶体学数据

Table 1 Crystal data of (MDPPF) $)_{2} \operatorname{Ir}($ acac)

\begin{tabular}{lclc}
\hline \multicolumn{1}{c}{ Item } & Value & Item & Value \\
\hline complex & $(\mathrm{MDPPF})_{2} \mathrm{Ir}(\mathrm{acac})$ & $F(000)$ & 842.0 \\
empirical formula & $\mathrm{C}_{39} \mathrm{H}_{29} \mathrm{~F}_{4} \mathrm{IrN}_{4} \mathrm{O}_{2}$ & $\mu / \mathrm{mm}^{-1}$ & 4.006 \\
formula weight & 853.88 & $T / \mathrm{K}$ & $293(2)$ \\
crystal color & orange & scan mode & multi-scan \\
crystal size/mm & $0.41 \times 0.23 \times 0.12$ & diffraction angle range $/\left(\mathrm{m}^{\circ}\right)$ & $2.77-26.37$ \\
crystal system & triclinic & reflections collected & 28168 \\
space group & $P \overline{1}$ & independent reflections & 6921 \\
$(\alpha, b, c) / \mathrm{nm}$ & $1.13984(3), 1.26718(3), 1.29541(3)$ & observable diffraction point & 6451 \\
$(\alpha, \beta, \gamma) /\left({ }^{\circ}\right)$ & $93.7181(19), 101.638(2), 110.853(3)$ & $R, w R$ & $0.0239,0.0599$ \\
$V / \mathrm{nm}^{3}$ & $1.69336(7)$ & parameter $(S)$ & 1.093 \\
$Z$ & 2 & $D_{\text {calc }} /\left(\mathrm{g} \cdot \mathrm{cm}^{-3}\right)$ & 1.675 \\
\hline
\end{tabular}

表 2 配合物(MDPPF) $)_{2} \operatorname{Ir}($ acac) 的主要的键长 $(l)$ 和键角 $(\theta)$ Table 2 Selected bond lengths $(l)$ and bond angles $(\theta)$ of the complex (MDPPF) $)_{2} \operatorname{Ir}($ acac)

\begin{tabular}{lclc}
\hline \multicolumn{1}{c}{ Bond } & $l / \mathrm{nm}$ & \multicolumn{1}{c}{ Bond } & $\theta /\left(^{\circ}\right)$ \\
\hline $\mathrm{I} 1-\mathrm{O} 1$ & $2.159(2)$ & $\mathrm{O} 1-\mathrm{Ir} 1-\mathrm{O} 2$ & $87.85(8)$ \\
$\mathrm{I} 1-\mathrm{O} 2$ & $2.145(2)$ & $\mathrm{N} 1-\mathrm{Ir} 1-\mathrm{O} 1$ & $95.17(9)$ \\
$\mathrm{I} 1-\mathrm{N} 1$ & $2.017(2)$ & $\mathrm{N} 1-\mathrm{I} 1-\mathrm{O} 2$ & $84.87(9)$ \\
$\mathrm{I} 1-\mathrm{N} 3$ & $2.024(2)$ & $\mathrm{C} 17-\mathrm{I} 1-\mathrm{O} 1$ & $175.05(10)$ \\
$\mathrm{I} 1-\mathrm{C} 17$ & $1.985(3)$ & $\mathrm{C} 17-\mathrm{Ir} 1-\mathrm{N} 3$ & $98.74(11)$ \\
$\mathrm{I} 1-\mathrm{C} 34$ & $1.989(3)$ & $\mathrm{C} 17-\mathrm{Ir} 1-\mathrm{C} 34$ & $88.59(12)$ \\
$\mathrm{F} 1-\mathrm{C} 8$ & $1.366(4)$ & $\mathrm{N} 2-\mathrm{C} 2-\mathrm{C} 1$ & $120.1(3)$ \\
$\mathrm{F} 2-\mathrm{C} 15$ & $1.359(4)$ & $\mathrm{N} 2-\mathrm{C} 2-\mathrm{C} 3$ & $117.5(3)$ \\
$\mathrm{F} 3-\mathrm{C} 35$ & $1.370(3)$ & $\mathrm{N} 2-\mathrm{C} 4-\mathrm{C} 11$ & $121.5(3)$ \\
\hline
\end{tabular}

相邻的原子构成一个不规则的八面体.

\section{2 光谱分析}

配体(MDPPF) 和配合物 (MDPPF) $2 \operatorname{Ir}(\mathrm{acac})$ 的紫
外-可见吸收光谱如图 2 所示. 在配体的紫外-可见吸 收光谱中, 226, 272 和 $312 \mathrm{~nm}$ 处有 3 个吸收峰, 可归 属为配体分子中 $\left(\pi-\pi^{*}\right)$ 和 $\left(n-\pi^{*}\right)$ 跃迁的吸收. 在配 合物的吸收曲线上, 在 227,276 和 $332 \mathrm{~nm}$ 处出现 3 个吸收峰, 可归属为自旋允许的 ${ }^{1} \mathrm{LC}$ 跃迁的吸收. 此 外, 在 379 和 $486 \mathrm{~nm}$ 处还有两个吸收峰, 可以分别 归属于配合物的 ${ }^{1} \mathrm{MLCT}$ (金属到配体的电荷跃迁) 和 ${ }^{3} \mathrm{MLCT}$ 的吸收峰.

在 $25^{\circ} \mathrm{C}$ 下，二氯甲烷溶液中 $(\mathrm{MDPPF})_{2} \operatorname{Ir}(\mathrm{acac})$ 的光致发光光谱如图 2 所示, 可以看出, 配合物 $(\mathrm{MDPPF})_{2} \operatorname{Ir}(\mathrm{acac})$ 的发射峰波长在 $555 \mathrm{~nm}$. 从特征 的吸收峰和无特征的发射峰以及较小的斯托克位 移 $(67 \mathrm{~nm})$ 可以推断出, $555 \mathrm{~nm}$ 处的发光主要来自金 属配合物 ${ }^{3} \mathrm{MLCT}$ 的磷光发射. ${ }^{15}$ 与(MDPP $)_{2} \operatorname{Ir}(\mathrm{acac})^{23}$

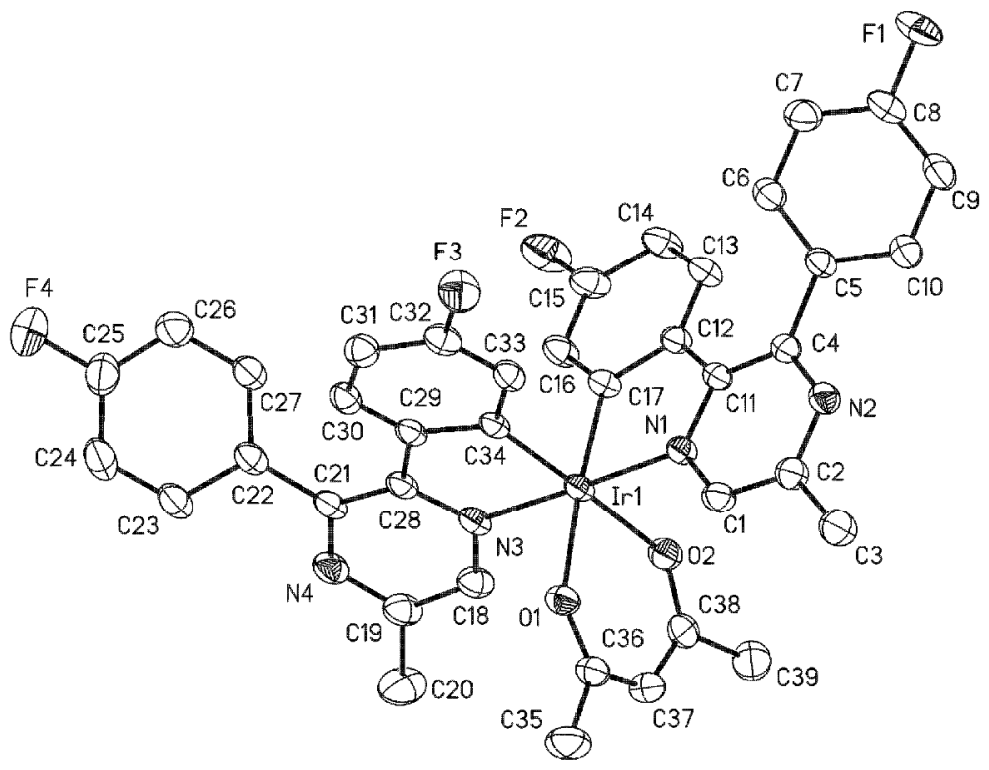

图 1 (MDPPF $)_{2} \operatorname{Ir}(\mathrm{acac})$ 的晶体结构图

Fig.1 Crystal structure of (MDPPF) $\operatorname{Ir}$ (acac) 


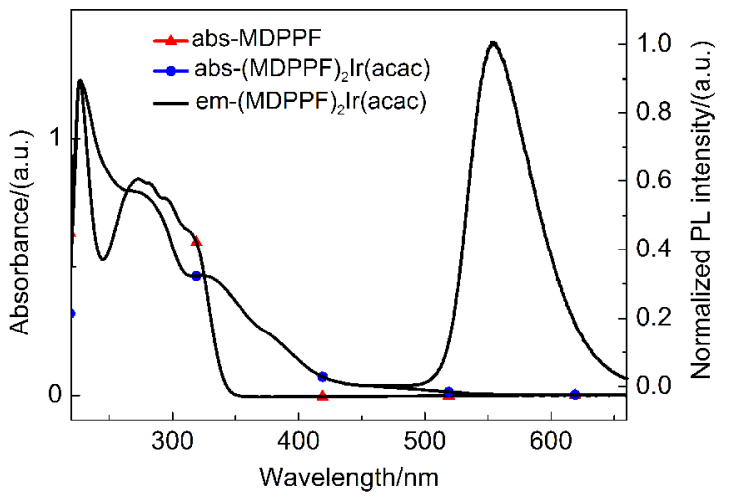

图 2 MDPPF 的紫外-可见吸收(abs)光谱和在 $\mathrm{CH}_{2} \mathrm{Cl}_{2}$ 溶液 中 (MDPPF $)_{2} \operatorname{Ir}(\mathbf{a c a c})$ 的紫外-可见吸收和发射(em)光谱

Fig.2 UV-Vis absorption (abs) spectrum of MDPPF and UV-Vis absorption spectra and emission (em) spectra of (MDPP) $)_{2} \operatorname{Ir}(\mathrm{acac})$ in $\mathrm{CH}_{2} \mathrm{Cl}_{2}$ solution

相比, 其发射波长蓝移了 $27 \mathrm{~nm}$, 原因是由于在苯环 中引入强吸电子的氟原子, 会降低配合物的 HOMO 能级, 使得配合物的 HOMO 与 LUMO 能隙变 大, 使发光峰蓝移.

\section{3 电致发光性能分析}

为了表征(MDPPF $)_{2} \operatorname{Ir}(\mathrm{acac})$ 的电致发光特性, 以 (MDPPF) $\operatorname{Ir}(\mathrm{acac})$ 为客体材料制备了结构为 ITO/NPB $(40 \mathrm{~nm}) / \mathrm{CBP}:(\mathrm{MDPPF})_{2} \operatorname{Ir}(\mathrm{acac})(5 \% / 7 \% / 10 \% / 15 \%, 20$ $\mathrm{nm}) / \mathrm{TPBi}(10 \mathrm{~nm}) / \mathrm{Alq}_{3}(30 \mathrm{~nm}) / \mathrm{LiF}(1 \mathrm{~nm}) / \operatorname{Al}(100 \mathrm{~nm})$ 的器件 (如图 3 所示). 器件中 NPB 作为空穴传输材 料, CBP 作为主体材料, TPBi 作为空穴阻挡材料和 电子传输材料, $\mathrm{Alq}_{3}$ 作为电子传输材料.

配合物 (MDPPF) $)_{2} \operatorname{Ir}(\mathrm{acac})$ 在不同电压驱动下器 件的电致发光光谱如图 4 所示. 器件的电致发光峰 均位于 $558 \mathrm{~nm}$, 我们没有观察到空穴传输层 NPB 或 者电子传输层 $\mathrm{Alq}_{3}$ 的发射, 表明 $\mathrm{TPBi}$ 起到了空穴阻 挡和电子传输的作用, 空穴和电子能够同时有效地 注入到发光层中. 另外, 我们也没有观察到 CBP 的 特征发射峰, 这说明主客体之间能够进行完全的能 量转移, 进而产生较强的磷光发射. 电致发光峰的 位置在 8-14 V 的电压范围内基本不随驱动电压的 变化而变化, 色坐标(CIE)基本稳定在 $(0.47,0.52)$, 属 黄光区域, 表明器件发光性能比较稳定. 电致发光 光谱与磷光材料的光致发光光谱基本一致, 但是 $\mathrm{EL}$ 相对于 PL, 右侧肩峰突起, 这个可能是三重态的配 体为中心的跃迁 $\left({ }^{3} \mathrm{LC}\right)$ 的发射增强所致. 文献中曾报 道过类似现象, 原因可能是在电场作用下配合物和 主体材料的相互作用引起的, ${ }^{22}$ 另外, 分子间发光也 可能导致长波部分的发射增强. ${ }^{32}$ 图 5 为电压为 $8 \mathrm{~V}$

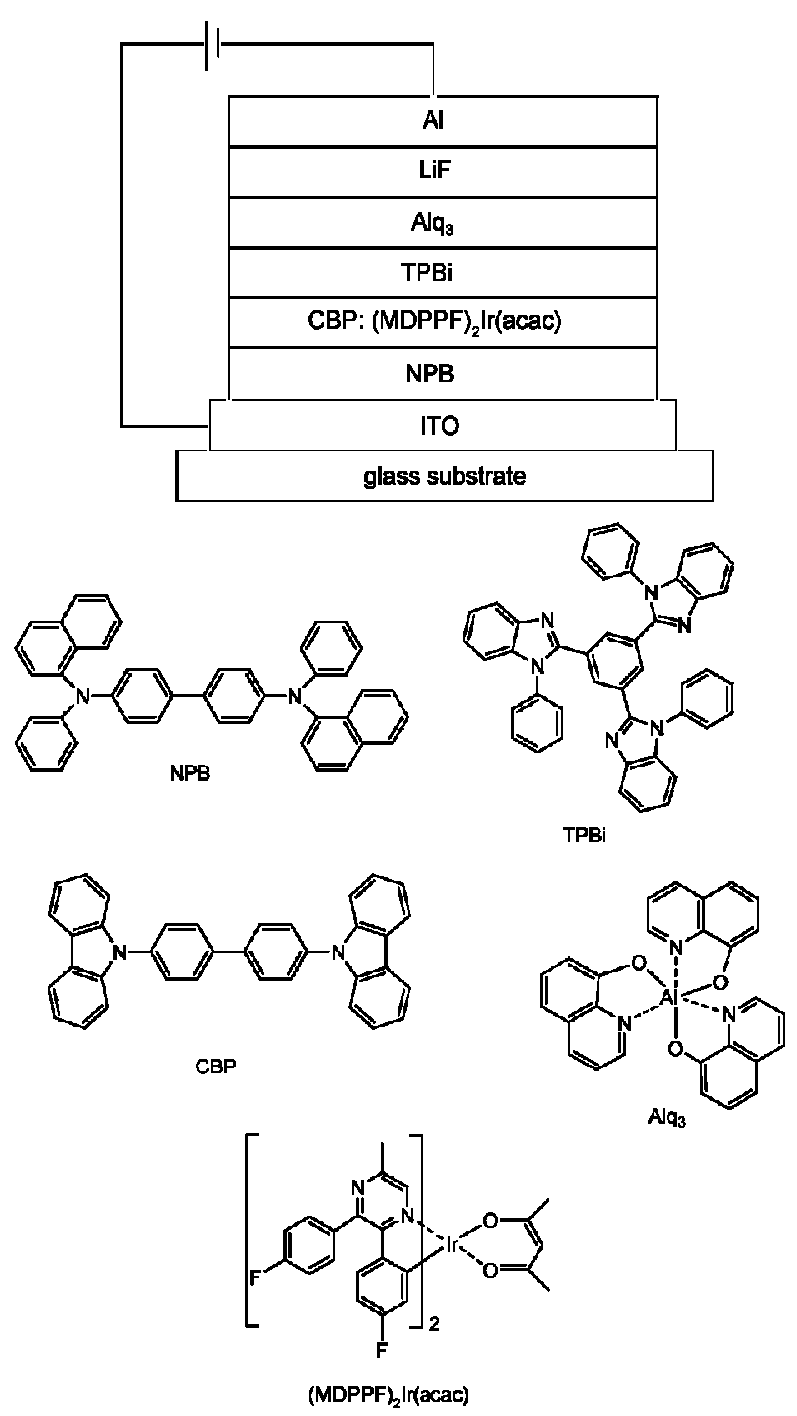

图 3 器件结构及所用材料的分子结构

Fig.3 Device configurations and molecular structures of the materials

ITO: indium-tin oxide

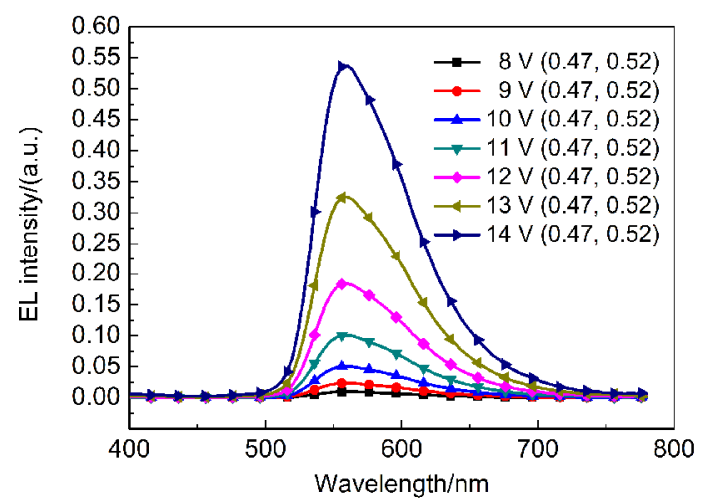

图 4 掺杂 $7 \%(w)$ 的器件在不同电压下的电致 发光光谱及色坐标

Fig.4 EL spectra of device and CIE coordinates at dopant concentration of $7 \%(w)$ at different voltages CIE: Internation Commission on Illumination 


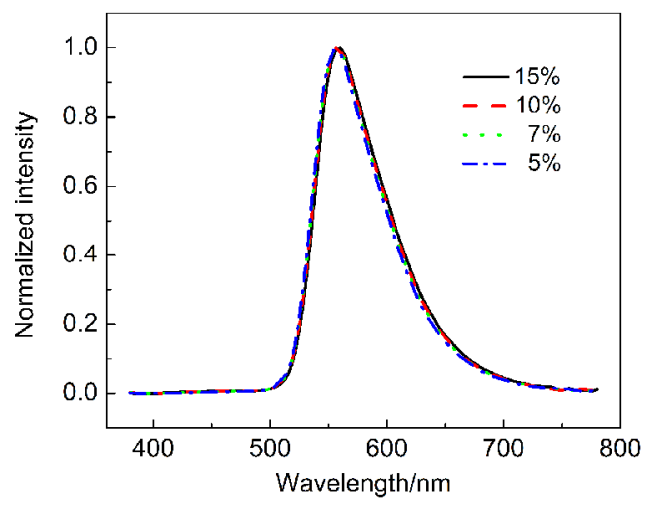

图 5 不同掺杂浓度 $(w)$ 在 $8 \mathrm{~V}$ 时的电致发光光谱

Fig.5 EL spectra of device under $8 \mathrm{~V}$ at different dopant concentrations $(w)$

时不同掺杂浓度的器件的 EL 光谱, 不同掺杂浓度 下的 EL 光谱基本相同, 说明长波部分的发射增强 不是来自于分子间发光.

表 3 是不同掺杂浓度的器件性能参数, 图 6 是 器件的电压 - 电流密度-亮度 $(V-J-L)$ 特性曲线. 从 表 3 和图 6 可以看出, 当电压达到 $4.0 \mathrm{~V}$ 左右时, 器件 有明显的黄光发射, 电流密度和亮度随电压的增大 而增大, 当驱动电压达到 $14.5 \mathrm{~V}$ 时, 掺杂浓度为 $10 \%$

表 3 不同掺杂浓度 $(w)$ 的器件性能参数

Table 3 Device performance based on different dopant concentrations $(w)$

\begin{tabular}{rrrrrrrrr}
\hline$w$ & $V_{\text {on }} / \mathrm{V}$ & $\frac{L_{\max }}{\left(\mathrm{cd} \cdot \mathrm{m}^{-2}\right)}$ & $\frac{\mathrm{CE}_{\max }}{\left(\mathrm{cd} \cdot \mathrm{A}^{-1}\right)}$ & $\frac{\mathrm{PE}_{\max }}{\left(1 \mathrm{~m} \cdot \mathrm{W}^{-1}\right)}$ & $\frac{\mathrm{CE}^{\mathrm{a}}}{\left(\mathrm{cd} \cdot \mathrm{A}^{-1}\right)}$ & $\frac{\mathrm{CE}^{\mathrm{b}}}{\left(\mathrm{cd} \cdot \mathrm{A}^{-1}\right)}$ & $\begin{array}{c}\mathrm{CIE} \\
\text { coordinate }\end{array}$ \\
\hline $15 \%$ & 3.9 & 28120 & 32.2 & 14.0 & 32 & 24 & $(0.47,0.52)$ \\
$10 \%$ & 3.8 & 32700 & 28.7 & 10.2 & 28 & 26 & $(0.47,0.52)$ \\
$7 \%$ & 4.1 & 29830 & 44.3 & 20.7 & 44 & 31 & $(0.47,0.52)$ \\
$5 \%$ & 4.0 & 31750 & 38.0 & 13.3 & 37 & 34 & $(0.47,0.52)$ \\
\hline
\end{tabular}

$V_{\text {on }}$ : turn-on voltage; $L_{\max }$ : maximum brightness; $\mathrm{CE}_{\max }$ : maximum current efficiency; $\mathrm{PE}_{\max }$ : maximum power efficiency; $\mathrm{CE}^{\mathrm{a}}$ : current efficiency at $1000 \mathrm{~cd} \cdot \mathrm{m}^{-2} ; \mathrm{CE}^{\mathrm{b}}$ : current efficiency at $10000 \mathrm{~cd} \cdot \mathrm{m}^{-2}$

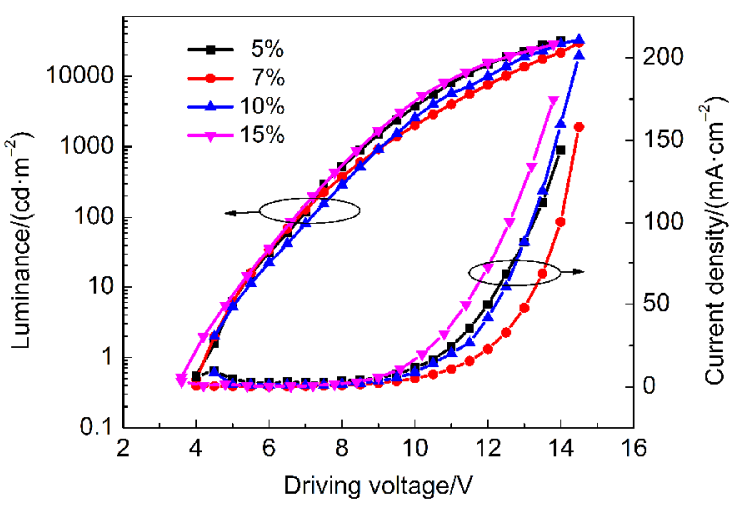

图 6 不同掺杂浓度器件的电压-电流密度-亮度图

Fig.6 $V-J-L$ curves of devices based on different dopant concentrations

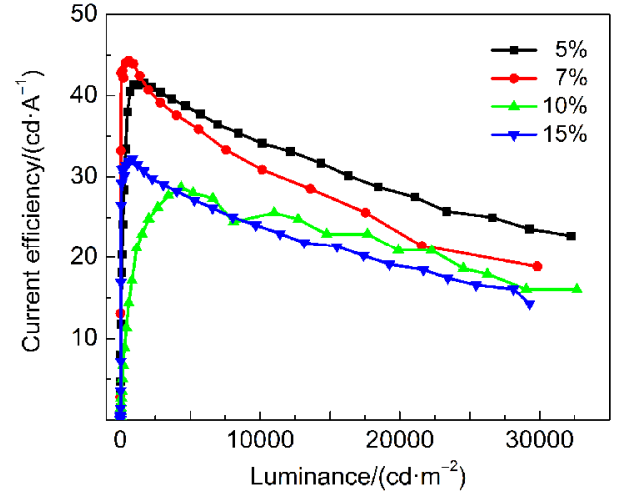

图 7 不同掺杂浓度器件的亮度-电流效率图

Fig.7 Luminance-current efficiency characteristics of devices based on different dopant concentrations

的器件的最大亮度可达 $32700 \mathrm{~cd} \cdot \mathrm{m}^{-2}$.

图 7 是不同掺杂浓度器件的亮度-电流效率曲 线. 可以看出高亮度下掺杂浓度低的器件具有更高 的效率, 这是因为低浓度发光中心降低了三重态-三 重态湮灭发生的概率. 而在 7\%掺杂浓度时, 器件在 最低亮度附近有最高电流效率 $44.3 \mathrm{~cd} \cdot \mathrm{A}^{-1}$, 功率效 率 $20.7 \mathrm{~lm} \cdot \mathrm{W}^{-1}$.

\section{4 结 论}

成功合成了一种新型蓝色磷光二嗪铱配合物 $(\mathrm{MDPPF})_{2} \mathrm{Ir}(\mathrm{acac})$, 并利用 $\mathrm{X}$ 射线单晶衍射仪测定了 该化合物的晶体结构. (MDPPF $)_{2} \operatorname{Ir}(\mathrm{acac})$ 的单晶结构 属于三斜晶系, $P \overline{1}$ 空间群, 在二氯甲烷溶液中呈黄 光发射, 发射峰为 $555 \mathrm{~nm}$. 制备了基于 (MDPPF $)_{2} \mathrm{Ir}$ (acac)的器件, 器件的发射峰位于 $551 \mathrm{~nm}$, 最大亮度 达到 $32700 \mathrm{~cd} \cdot \mathrm{m}^{-2}$, 最大电流效率为 $44.3 \mathrm{~cd} \cdot \mathrm{A}^{-1}$, 最 大功率效率为 $20.7 \mathrm{~lm} \cdot \mathrm{W}^{-1}$, 色坐标保持在 $(0.47$, $0.52)$. (MDPPF $)_{2} \operatorname{Ir}(\mathrm{acac})$ 是一种性能优良的黄色磷光 材料, 可用于制备高效的黄色电致磷光器件, 或与 蓝光材料复合用于制备白光发光器件.

\section{References}

(1) Baldo, M. A.; O'Brien, D. F.; You, Y.; Shoustikov, A.; Sibley, S.; Thompson, M. E.; Forrest, S. R. Nature 1998, 395 (6698), 151. doi: $10.1038 / 25954$

(2) Chi, Y.; Chou, P. T. Chem. Soc. Rev. 2007, 36 (9), 1421. doi: 10.1039/b608951h

(3) Carlson, B.; Phelan, G. D.; Kaminsky, W.; Dalton, L.; Jiang, X Z.; Liu, S.; Jen, A. Y. J. Am. Chem. Soc. 2002, 124 (47), 14162

(4) Cheng, Y. M.; Yeh, Y. S.; Ho, M. L.; Chou, P. T. Inorg. Chem. 2005, 44 (13), 4594. doi: 10.1021/ic0505347

(5) Wu, P. C.; Yu, J. K.; Song, Y. H.; Chi, Y.; Chou, P. T.; Peng, S. 
M.; Lee, G. H. Organometallics 2003, 22 (24), 4938. doi: 10.1021/om034037e

(6) Moussa, J.; Wong, K. M. C.; Chamoreau, L. M.; Amouri, H.; Yam, V. W. W. Dalton. Trans. 2007, No. 32, 3526.

(7) Kavitha, J.; Chang, S. Y.; Chi, Y.; Yu, J. K.; Hu, Y. H.; Chou, P. T.; Peng, S. M.; Lee, G. H.; Tao, Y. T.; Chien, C. H.; Carty, A. J. Adv. Funct. Mater. 2005, 15 (2), 223.

(8) Brooks, J.; Babayan, Y.; Lamansky, S.; Djurovich, P. I.; Tsyba, I.; Bau, R.; Thompson, M. E. Inorg. Chem. 2002, 41 (12), 3055. doi: $10.1021 / \mathrm{ic} 0255508$

(9) Graham, K. R.; Yang, Y. X.; Sommer, J. R.; Shelton, A. H.; Schanze, K. S.; Xue, J. G.; Reynolds, J. R. Chem. Mater. 2011, 23 (24), 5305. doi: 10.1021/cm202242x

(10) Wiegmann, B.; Jones, P. G.; Wagenblast, G.; Lennartz, C.; Münster, I.; Metz, S.; Kowalsky, W.; Johannes, H. H. Organometallics 2012, 31 (15), 5223. doi: 10.1021/om300458f

(11) Gong, X.; Ostrowski, J. C.; Bazan, G. C.; Moses, D.; Heeger, A. J.; Liu, M. S.; Jen, A. K. Y. Adv. Mater. 2003, 15 (1), 45.

(12) Chen, X.; Liao, J. L.; Liang, Y. M.; Ahmed, M. O.; Tseng, H. E.; Chen, S. A. J. Am. Chem. Soc. 2003, 125 (3), 636. doi: 10.1021/ ja0211151

(13) Tsuboyama, A.; Iwawaki, H.; Furugori, M.; Mukaide,T.; Kamatani, J.; Igawa, S.; Moriyama, T.; Miura, T.; Takiguchi, T.; Okada, S.; Hoshino, M.; Ueno, K. J. Am. Chem. Soc. 2003, 125 (42), 12971. doi: 10.1021/ja034732d

(14) Lamansky, S.; Djarovich, P.; Murphy, D.; Feras, A. R.; Lee, H. E.; Adachi, C.; Burrows, P. E.; Forrest, S. R.; Mark, E. J. Am. Chem. Soc. 2001, 123 (18), 4304. doi: 10.1021/ja003693s

(15) Hwang, F. M.; Chen, H. Y.; Chen, P. S.; Liu, C. S.; Chi, Y.; Shu, C. F.; Wu, F. L.; Chou, P. T.; Peng, S. M.; Lee, G. H. Inorg. Chem. 2005, 44 (5), 1344.

(16) Su, Y. J.; Hung, H. L.; Li, C. L.; Chien, C. H.; Tao, Y. T.; Chou, P. T.; Datta, S.; Liu, R. S. Adv. Mater. 2003, 15 (11), 884. doi: 10.1002/adma.200304630

(17) King, K. A.; Watts, R. J. J. Am. Chem. Soc. 1987, 109, 1589. doi: $10.1021 / \mathrm{ja} 00239 \mathrm{a} 060$

(18) Ayala, N. P.; Flynn, C. M., Jr.; Sacksteder, L.; Demas, J. N.; DeGraff, B. A. J. Am. Chem. Soc. 1990, 112, 3837. doi: 10.1021/ ja00166a018

(19) Polson, M.; Fracasso, S.; Bertolasi, V.; Ravaglia, M.; Scandola,
F. Inorg. Chem. 2004, 43, 1950. doi: 10.1021/ic0351848

(20) Guo, Y, H.; Mei, Q. B.; Yan, F.; Wang, L. X.; Weng, J. N.; Zhang, B.; Huang, W. Acta Phys. -Chim. Sin. 2012, 28 (4), 739. [ 郭远辉, 梅群波, 颜 芳, 王玲霞, 翁洁娜, 张 涁, 黄 维. 物理化学学报, 2012, 28 (4), 739.] doi: 10.3866/PKU. WHXB201112222

(21) Holmes, R. J.; Forrest. S. R.; Tung, Y. J.; Kwong, R. C.; Brown, J. J.; Garon, S.; Thompson, M. E. Appl. Phys. Lett. 2003, 82 (15), 2422. doi: $10.1063 / 1.1568146$

(22) Duan, J. P.; Sun, P. P.; Cheng, C. H. Adv Mater. 2003, 15 (3), 224. doi: 10.1002/adma.200390051

(23) Liu, Z. W.; Min, G.; Bian, Z. Q.; Nie, D. B.; Gong, Z. L.; Li, Z. B.; Huang, C. H. Adv. Funct. Mater. 2006, 16 (11), 1441.

(24) Ge, G. P.; He, J.; Guo, H. Q.; Wang, F. Z.; Zou, D. C. J. Organomet. Chem. 2009, 694 (19), 3050. doi: 10.1016/j. jorganchem.2009.05.037

(25) Zhang, G. L.; Liu, Z. H.; Guo, H. Q.; Chuo, Y. T.; Zhen, C. G.; Zou, D. C. Chem. J. Chin. Univ. 2004, 25 (3), 397. [张国林, 刘泽华, 郭海清, 啜玉涛, 甄常刮, 邹德春. 高等学校化学学报, 2004, 25 (3), 397.]

(26) Zhang, G. L.; Guo, H. Q.; Chuo, Y. T.; Zou, D. C. Acta Chim. Sin. 2005, 63 (2), 143. [张国林, 郭海清, 啜玉涛, 邹德春. 化 学学报, 2005, 63 (2), 143.]

(27) Zhang, G. L.; Liu, Z. H.; Guo, H. Q. Acta Phys. -Chim. Sin 2003, 19 (10), 889. [张国林, 刘泽华, 郭海清. 物理化学学报, 2003, 19 (10), 889.] doi: 10.3866/PKU.WHXB20031001

(28) Zhang, G. L.; Guo, H. Q.; Chuo, Y. T.; Zou, D. C. Materials Letters 2005, 59 (24-25), 3002. doi: 10.1016/j. matlet.2005.05.004

(29) Ho, C. L; Wong, W. Y.; Wang, Q.; Ma, D. G.; Wang, L.; Lin, Z. Y. Adv. Funct. Mater. 2008, 18 (6) , 928.

(30) Wang, Y.; Herron, N.; Grushin, V. V.; LeClouxand, D.; Petrov, V. Appl. Phys. Lett. 2001, 79 (4), 449. doi: 10.1063/1.1384903

(31) Ge, G. P.; Zhang, G. L.; Guo, H. Q.; Chuo, Y. T.; Zou, D. C. Inorg. Chim. Acta 2009, 362 (7), 2231. doi: 10.1016/j. ica.2008.10.001

(32) Wang, J.; Zhang, F. J.; Xu, Z.; Wang, Y. S. Acta Phys. -Chim Sin. 2012, 28 (4), 949 . [王 建, 张福俊, 徐征, 王永生. 物理化学学报, 2012, 28 (4), 949.] 